\title{
The stability and degradation kinetics of acetylsalicylic acid in different organic solutions revisited - an UHPLC-ESI-QTOF spectrometry study
}

\author{
Robert Skibinski, Lukasz Komsta*
}

Department of Medicinal Chemistry, Pharmaceutical Faculty, Medical University of Lublin, Jaczewskiego 4, 20-090 Lublin, Poland

\begin{tabular}{l} 
ARTICLE INFO \\
\hline Received 02 November 2015 \\
Accepted 15 December 2015
\end{tabular}

\section{Keywords:}

acetylsalicylic acid,

stability,

solutions,

UHPLC,

ESI,

Q-TOF.

\begin{abstract}
Ultra high performance liquid chromatography (UHPLC), coupled with accurate quadrupole-time-of-flight (Q-TOF) mass spectrometry, was used for the stability study of acetylsalicylic acid within a variety of different organic solutions: methanol, ethanol, propan-2-ol, acetonitrile, tetrahydrofuran and 1,4-dioxane. With the use of gradient elution chromatography and mass spectrometry detection in negative ionization, MS and MS/MS spectra were recorded simultaneously. In addition, quantitative, as well as qualitative analysis was performed during one assay. The stability of acetylsalicylic acid in such solutions was tested at room temperature, in a $12 \mathrm{~h}$ period. In the work, in all cases, only one main degradation product, salicylic acid, was found. What is more, the work revealed that the degradation of aspirin in the tested organic solutions yields apparent second-order kinetics. The study also demonstrated that acetonitrile and 1,4-dioxane turned out to be the most stable solvents, and an above $80 \%$ of initial concentration of acetylsalicylic acid was found in this case. Furthermore, the most popular analytical solvents, methanol and ethanol, were found to be very unstable media. Herein, below $40 \%$ of initial concentration of acetylsalicylic acid was seen after $12 \mathrm{~h}$. The obtained results were also compared with the degradation of acetylsalicylic acid in a water solution. In this situation, only about $25 \%$ of the analyzed compound was resolved to salicylic acid in the same time frame.
\end{abstract}

\section{INTRODUCTION}

Acetylsalicylic acid, also known as 'aspirin', is a very popular analgesic and antipyretic agent that has very labile properties. Hence, knowledge concerning the degradation of this drug as placed in a variety of solutions, is important from the point of view of analytical procedures. This because the usage of an improper solvent for the preparation of aspirin samples could lead to its fast degradation, and, hence, introduce into such work, significant analytical errors [3]. Although it can seem to the reader that the stability of acetylsalicylic acid is a well elaborated topic, very few studies can actually be found in current literature. Most often, the stability of this agent was tested in mixtures of water and polar solvents. Gharbo and Williamson, for example, noticed its degradation in aqueous ethanol solution [3], while Chang and Whitworth [2] studied the degradation of aspirin in water-propylene glycol and water-triethylene glycol diacetate mixtures. Moreover, various substituted and nonsubstituted polyhydric alcohols, in the absence and presence of water, were tested by Jun and co-workers [4]. Furthermore, the hydrolysis of the acid in water-polyethylene glycol 400, water-methanol-acetic acid, phosphate buffer and freshly drawn blood and plasma was also investigated by Bakar and Niazi [1]. In addition, the stability of aspirin was also studied in mixed solvent systems (ethanol, ether, glycols and water) under ultrasonic conditions [5]. The aim of our study was, hence, to establish the most stable solvent for routine analytical procedures. For this purpose, a new analytical method which employed a new generation hybrid Q-TOF spectrometer combined with a UHPLC system, was developed.

\footnotetext{
* Corresponding author

e-mail: lukasz.komsta@umlub.pl
} 


\section{MATERIALS AND METHODS}

\section{Materials}

For our study, acetylsalicylic acid (99.5\% purity) was obtained from BUFA B.V. (Uitgeest, Holland). Formic acid for LC-MS was purchased from Fluka (Steinherin, Germany). Solvents (1,4-dioxane, acetonitrile, tetrahydrofuran, propan-2-ol, water, methanol, ethanol) were purchased from Merck (Dramstadt, Germany). A stock solution of acetylsalicylic acid was prepared in methanol at a concentration of $1 \mathrm{mg} / \mathrm{mL}$.

\section{UHPLC-ESI-Q-TOF analysis}

UHPLC-MS/MS analysis was performed with the use of a Agilent Accurate-Mass Q-TOF LC/MS G6520B system, with a dual electrospray (DESI) source, an Infinity 1290 UHPLC system and a Zorbax Extend-C18 (2.1x50mm, $\mathrm{dp}=1.8 \mu \mathrm{m}$ ) column (Agilent, Santa Clara, USA). A mixture of acetonitrile (A) and water (B), with the addition of a $0.1 \%$ solution of formic acid in both media was used as a mobile phase. The gradient elution was carried out at a constant flow of $0.4 \mathrm{ml} / \mathrm{min}$, from $15 \% \mathrm{~A}(85 \% \mathrm{~B})$ to $80 \% \mathrm{~A}$, in a time period of $0-13$ mins and with a $1 \mathrm{~min}$ isocratic post-time (15\%A). The injection volume was $4 \mu \mathrm{l}$ and the column temperature was maintained at $25^{\circ} \mathrm{C}$. The instrument condition optimization was initiated through the proper tuning of the Q-TOF detector while in a negative mode, and with the use of an Agilent ESI-L tuning mix in a high resolution mode. Here, the following settings were applied: gas temp.: $250^{\circ} \mathrm{C}$, drying gas: $10 \mathrm{l} / \mathrm{min}$, nebulizer pressure: 40 psig, capillary voltage: $3500 \mathrm{~V}$, fragmentor voltage: $110 \mathrm{~V}$, skimmer voltage: $65 \mathrm{~V}$, octopole voltage: $250 \mathrm{~V}$. Data acquisition was performed in the auto MS/MS mode, the spectral parameters being: mass range: $50-1100 \mathrm{~m} / \mathrm{z}$ and acquisition rate: $1.4 \mathrm{spectra} / \mathrm{s}$. Furthermore, collision energy was calculated by way of auto algorithm, utilizing the formula: $4.6 \mathrm{~V}$ (slope)*(m/z)/100 $+4.8 \mathrm{~V}$ (offset). Mass 122.985587 and 1033.988109 were used as lock masses.

\section{Quantitative analysis and degradation kinetics}

The method calibration for the determination of the concentration of acetylsalicylic acid in the tested samples was performed via the use of MS detection. Herein, the extracted ion chromatograms (EIC) were set for mass $179.0349 \mathrm{~m} / \mathrm{z}$, and a calibration curve was obtained in the range: $0.4-14$ $\mu \mathrm{g} / \mathrm{mL}$. This was studied by fitting the results to linear leastsquares regression. All calibration standards were analyzed five times, subsequently, the average calibration curve with its statistic parameters was calculated. The obtained calibration curve was then used for the determination of the degradation kinetics of acetylsalicylic acid within the various solvents. Working solutions prepared in the tested solvents were stored at room temperature $\left(21^{\circ} \mathrm{C}\right)$ and analyzed by the UHPLC-ESI-Q-TOF system in the times of: 0, 2, 4, 6, 8,10 and 12 hour post-preparation. The degradation kinetic parameters: rate constant $(\mathrm{k})$ and $\mathrm{t}_{1 / 2}$ were calculated with the use of the standard equation:

\section{RESULTS AND DISCUSSION}

In order to achieve high selectivity by way of utilizing the developed method, MS detection was employed for all quantification, while DAD detection was only used for the additional chromatographic process put in place for monitoring purposes (Fig. 1). After chromatographic separations, the analyzed compounds were identified through the use of TOF accurate MS spectra, as well as fragmentation MS/MS spectra. Acetylsalicylic acid was encountered in all analyzed samples and its molecular ion $(179.0349 \mathrm{~m} / \mathrm{z})$ was detected with very good accuracy (1.22 ppm). The obtained MS/MS spectra also confirmed its structures (Fig. 2A). In all tested solvents, one main degradation product, salicylic acid $(137.0242 \mathrm{~m} / \mathrm{z})$, was found - this, also with good accuracy (1.82 ppm). What is more, its MS/MS spectra were registered (Fig. 2B). The registered data from Q-TOF was further enlisted for the quantification of aspirin in all solvents by way of utilizing EIC $(179.0349 \mathrm{~m} / \mathrm{z})$. The calibration of the quantitative analysis method was performed in the range $0.4-14 \mu \mathrm{g} / \mathrm{mL}$. The obtained calibration curve: $y=18697.30( \pm 456.34) x+6971.73( \pm 1961.48)$ was linear over the concentration range $(r=0.9991)$ and the limits of detection (LOD) and quantification (LOQ) were $0.04 \mu \mathrm{g} / \mathrm{mL}$ and $0.14 \mu \mathrm{g} / \mathrm{mL}$, respectively (experimentally obtained against signal to noise level). Herein, RSD values were in the range $0.14-1.83 \%$. The obtained results were employed for calculating the concentration of acetylsalicylic acid at set time intervals in all the tested organic solvents and in water. The degradation profile of aspirin in these solvents is shown in Fig. 3.
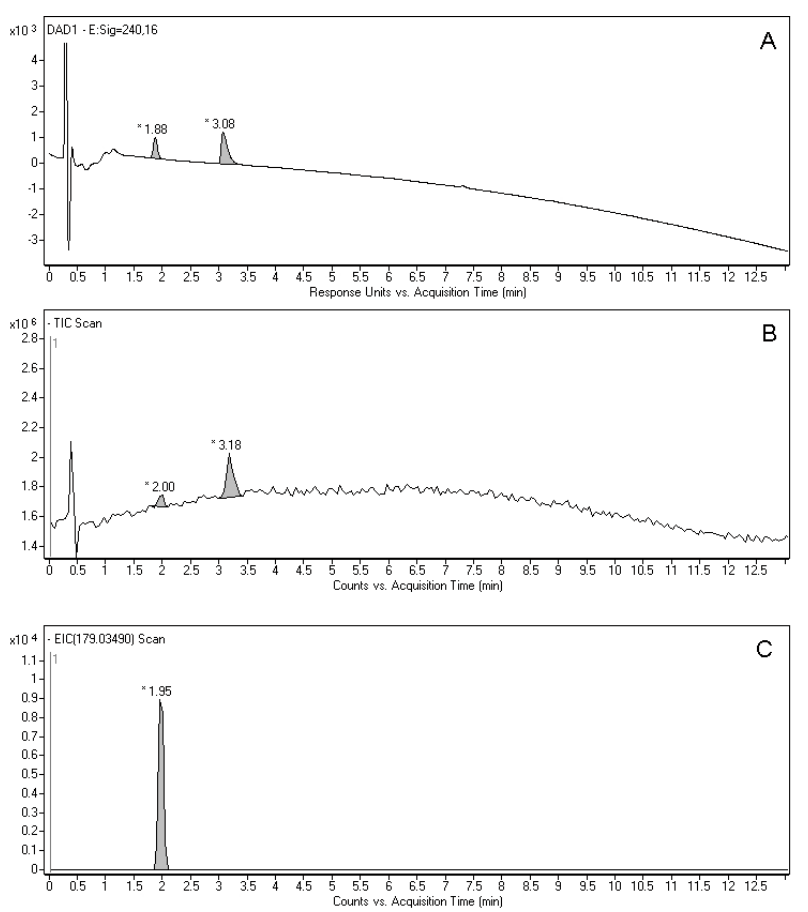

Figure 1. UHPLC-DAD (A), total ion (B) and extracted ion (C) chromatograms obtained for acetylsalicylic acid $\left(t_{R}=2 \mathrm{~min}\right)$ in methanol after $12 \mathrm{~h}$. Salicylic acid $\mathrm{t}_{\mathrm{R}}=3.1 \mathrm{~min}$.

$$
1 / \mathrm{c}=1 / \mathrm{c}_{0}-\mathrm{kt}
$$



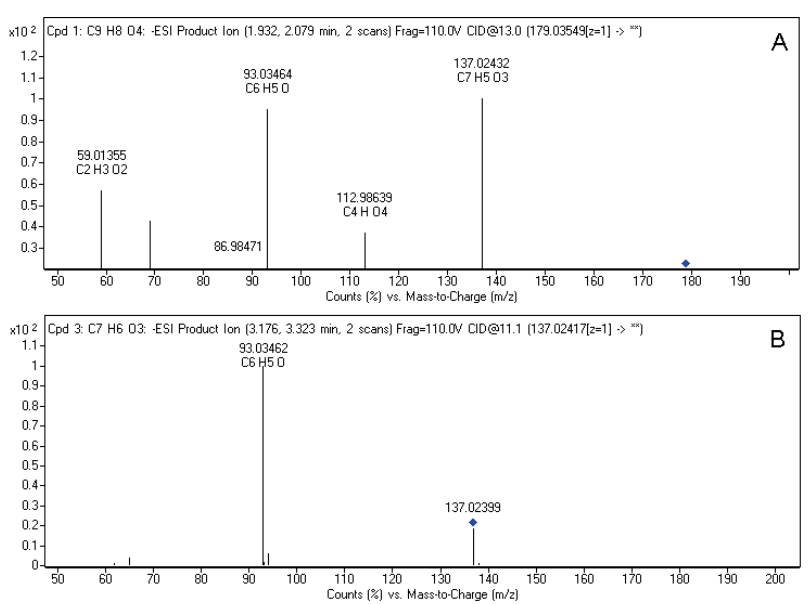

Figure 2. Q-TOF MS/MS spectra of acetylsalicylic acid (A) and salicylic acid (B) in methanol solvent

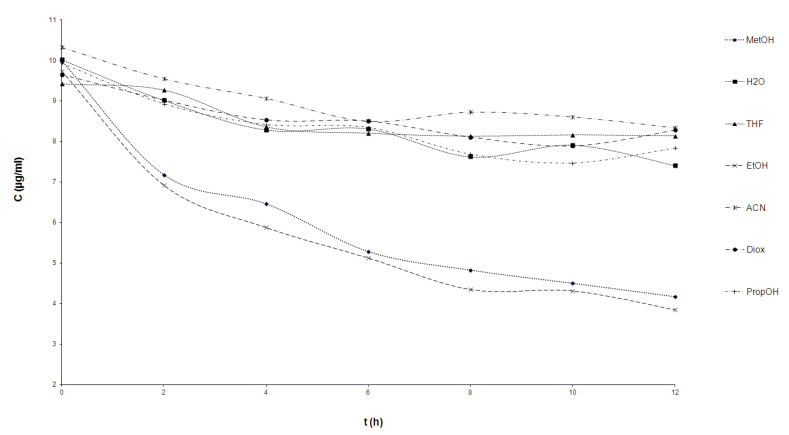

Figure 3. Degradation profile of acetylsalicylic acid in methanol $(\mathrm{MetOH})$, acetonitrile $(\mathrm{ACN})$, tetrahydrofuran (THF), ethanol (EtOH), 1,4-dioxane (Diox), propan-2-ol (PropOH) and water $\left(\mathrm{H}_{2} \mathrm{O}\right)$ solutions

The determined results were used to assign the kinetics reaction and the best fit was observed for the equation (1). This action suggested that the decomposition of acetylsalicylic acid in all the tested organic solvents yields an apparent second-order kinetics reaction. The rate constant values, the half-life times and the correlation coefficients of the degradation process for the analyzed compounds are presented in Table 1. In this work, 1,4-dioxane and acetonitrile turned out to be the most stable solvents, and above $80 \%$ of initial concentration of acetylsalicylic acid was found in both solvents after $12 \mathrm{~h}$ degradation. However, the most popularly utilized analytical solvents, methanol and ethanol, were revealed to be very unstable media. In both, only about $40 \%$ of the initial concentration of acetylsalicylic acid was found in the same degradation time. The obtained results regarding all solvents were also compared with the degradation of acetylsalicylic acid in a water solution (containing $1 \%$ of methanol from stock solution). In this solvent, up to about $70 \%$ of the aspirin was found after $12 \mathrm{~h}$. This shows that some of the popular organic solvents that are commonly utilized used in various analytical procedures are very hazardous for establishing accurate analytical assays of acetylsalicylic acid. This situation is even more so than for the well-known hydrolytic media - water.
Table 1. Degradation kinetics parameters in tested solvents

\begin{tabular}{|l|c|c|c|}
\hline \multicolumn{1}{|c|}{ Solvent } & $k\left(\mathrm{~h}^{-1}\right)$ & $t_{1 / 2}(\mathrm{~h})$ & $\mathrm{r}$ \\
\hline 1,4-dioxane & 0.00124 & 83.35 & 0.9011 \\
\hline Acetonitrile & 0.00153 & 63.37 & 0.9101 \\
\hline Tetrahydrofuran & 0.00166 & 62.14 & 0.8925 \\
\hline Propan-2-ol & 0.00243 & 41.43 & 0.9127 \\
\hline Water & 0.00262 & 38.09 & 0.9369 \\
\hline Methanol & 0.01135 & 8.83 & 0.9882 \\
\hline Ethanol & 0.01261 & 8.15 & 0.9853 \\
\hline
\end{tabular}

\section{CONCLUSIONS}

We established that the most stable solvents for acetylsalicylic acid were 1,4-dioxane and acetonitrile, with the half-life times of 83 and $63 \mathrm{~h}$, respectively. Furthermore, the fastest degradation of acetylsalicylic acid was observed in ethanol and methanol solutions, the half-life times being about $8 \mathrm{~h}$ in both cases. The obtained results of our work can be helpful in the process of developing new analytical methods of acetylsalicylic acid assay.

\section{ACKNOWLEDGEMENT}

The paper was developed with the use of the equipment purchased within the Project "The equipment of innovative laboratories doing research on new medicines used in the therapy of civilization and neoplastic diseases", within the Operational Program Development of Eastern Poland 2007-2013, Priority Axis I Modern Economy, Operations I.3 Innovation Promotion.

\section{REFERENCES}

1. Bakar S.K. and Niazi S., Stability of aspirin in different media. J. Pharm. Sci. 72, 1024, 1983.

2. Chang R.K. and Whitworth C.W., Aspirin degradation in mixed polar solvents, Drug Develop. Ind. Pharm., 10, 515, 1984.

3. Gharbo S.A. and Williamson M.J., Degradation of aspirin in solutions. Drug Develop. Ind. Pharm. 12, 927, 1986.

4. Jun H.W. et al, Aspirin stability in substituted and nonsubstituted polyhydric alcohols, in absence and presence of added water. $J$. Pharm. Sci. 63, 133, 1974.

5. Needham Jr, T.E. and Gerraughty, R.J., Ultrasonic degradation of aspirin in mixed solvent systems, J. Pharm. Sci., 58, 62, 1969. 